\title{
Memory psychophysics for visual area and length
}

\author{
STEPHEN M. KERST and JAMES H. HOWARD, JR. \\ Catholic University of America, Washington, D.C. 20064
}

\begin{abstract}
Independent groups of observers made magnitude estimates of geographical area or interstate distance. In Experiment 1, observers estimated the areas of nations or of states of the United States from memory. In Experiment 2, estimates of state area were made either with a map present or from memory after the map had been studied. Similarly, in Experiment 3, observers made perceptual or memorial estimates of interstate distances. Perceptual estimates of distance and geographical area were related to actual stimulus magnitude by power functions whose exponents were similar to those found with conventional procedures. Memory estimates were also related to actual area and distance by power functions. Comparison of memory and perceptual exponents showed that for both area and distance, the memory exponent was equal to the square of the perceptual exponent. The results of Experiment 3 were predicted by a "re-perceptual" model of memory for continuous dimensions, which was developed to describe the results of Experiment 2.
\end{abstract}

A number of recent empirical findings have emphasized the similarity of perceptual and cognitive processes (e.g., Shepard \& Podgorny, in press). This suggests that the well established techniques developed to investigate human psychophysical judgment may be fruitfully applied to the analysis of many cognitive processes as well. For example, Moyer (1973) has proposed that subjects make an "internal psychophysical judgment" among memory representations when they are asked to compare the sizes of two concrete objects from memory. As with perceptual judgments of stimuli that are actually presented (Curtis, Paulos, \& Rule, 1973), reaction time (RT) for memory comparisons is inversely related to the difference between the rated sizes of the objects. This effect has been called the symbolic distance effect (SDE) (Moyer \& Bayer, 1976; Moyer \& Dumais, in press).

Other investigators have replicated the basic finding that RT for memorial comparisons is lawfully related to the difference in subjective size ratings of the comparison objects (Jamieson \& Petrusic, 1975). Furthermore, Kerst and Howard (1977) found that subjects' size ratings predicted comparison RT more accurately than these size ratings expressed only as ranks. This result suggests that subjects use interval information about the degree to which objects differ

Since the contribution of the two authors was equivalent, the order of authorship is arbitrary. The authors acknowledge the helpful comments of Darlene V. Howard and Brian McLaughlin on an earlier version of this manuscript, and the contributions of Nick Geleta and Don Burgy in assisting with data analysis. Requests for reprints should be addressed to Stephen $M$. Kerst Boys Town Center for the Study of Youth Development, Catholic University, Washington, D. C. 20064. This research was supported in part by a grant from the National Science Foundation to the Catholic University. in rated size in addition to knowledge of the ordinal rankings of the stimuli on the size dimension. Paivio's (1975) finding that comparison RTs are negatively related to the ratios of the rated sizes of objects is also consistent with this interpretation. Moyer and Bayer (1976) separated the ordinal and interval components of the comparison process by asking observers to make size comparisons from memory for two series of circles differing in their range of absolute sizes (the small range had diameters of $11-17 \mathrm{~mm}$ and the large range had diameters of $11-23 \mathrm{~mm}$ ). The results revealed that both the ordinal and absolute size differences between comparison stimuli affected RT. Since this result would not be expected if observers' judgments were based only on the ordinal relations among the stimuli, the authors concluded that the absolute size of the circles is preserved in memory and that observers make use of this information when they make mental comparisons." As Shepard and Podgorny (in press) have pointed out, this similarity between memorial and perceptual comparisons suggests that the memory code activated by symbolically presented information has much in common with the perceptual experience of the actual objects themselves. In particular, memory representations, like percepts, have continuous characteristics in that they accurately reflect the relative subjective sizes of the objects they represent.

The present experiments were carried out in the spirit of Moyer's (1973) "internal psychophysics" to investigate the degree to which perceptual information on a continuous dimension such as size is preserved in memory. Psychophysicists have long been concerned with the relation between the magnitude of a sensation and the actual physical magnitude of the stimulus presented to the observer. In the present study, we examine the relation between actual stimulus magnitude 
and the stimulus magnitude represented in memory. It is well known that psychophysical judgments of size are systematically related to actual stimulus area, even though the range of actual physical magnitudes may be many times greater than the range of corresponding subjective estimates (e.g., Stevens, 1975; Teghtsoonian, 1965). For additive dimensions such as weight, brightness, sound intensity, and area, the relation between psychological magnitude $(\Psi)$ and physical magnitude $(\Phi)$ can be described by a power function of the following form:

$$
\Psi=\mathrm{k} \Phi^{\mathrm{n}}
$$

where $\mathrm{n}$ is a parameter that depends on the judgment continuum and $\mathrm{k}$ is a scaling factor that depends on the unit of measurement that is used. It can be shown rigorously (Luce, 1959) that this equation expresses a relationship between two ratio scales. Psychological scales of estimated sensation have been shown to have the properties of ratio scales; that is, equal stimulus ratios correspond to equal ratios of subjective magnitude estimates at all locations on the scale (e.g., Stevens, 1975). The first question addressed in the present study is whether a similar state of affairs exists in memory. That is, do estimates based on memory representations reveal the ratio properties of psychophysical scales for perceptual judgments, or is the memory scale more crude and inexact?

Some preliminary data addressing this issue have been reported by Björkman, Lundberg, and Tärnblom (1960) and Moyer, Bradley, and Cutcomb (Note 1). In the Björkman et al.(1960) study, observers were first required to learn arbitrary names (letters of the alphabet) for a series of circles of differing area or a series of objects of differing weight. After learning to name the stimuli, observers were asked to provide direct ratio estimates of the object areas or weights when only the name and a standard stimulus were presented. The authors examined the memory-psychophysical relation among perceived and remembered magnitudes and the corresponding physical values and concluded that "the power function seems to be an adequate description of the relationship between memory and percept" (Björkman et al., 1960, p. 136). Similarly, Moyer et al. (Note 1) used a direct magnitude estimation procedure to determine the memorypsychophysical functions for volume, weight, and brightness. Moyer's results were consistent with those of Björkman et al. in revealing that "the remembered value of a nominal stimulus can be well described as a power function of the physical value of its referent" (p. 7). His results further revealed that some of the modality. specific effects observed in perceptual judgments are preserved in the memorial judgments. For example, the exponents for brightness judgments were smaller than those for weight judgments, both for memorial and perceptual estimates.
In Experiments 1 and 2, the relation between remembered and actual size was examined by asking observers to estimate the sizes of familiar geometric shapes (states of the United States and foreign countries). Experiment 1 examined the memorypsychophysical relation when these judgments were made on the basis of general knowledge, whereas Experiment 2 investigated the relationship when observers were allowed to briefly study a map before making their judgments. In Experiment 3, predictions of two alternative theories, developed to account for the findings of the first two experiments, were tested.

\section{EXPERIMENT 1}

\section{Method}

Subjects. Twenty-six student volunteers judged the size of states from memory, and 16 other volunteers made similar judgments for countries.

Materials. Observers recorded their size estimates of the 48 states of the continental United States or of 48 countries of Europe and Asia on computer-generated sheets. A different random order of states or countries was used for each participant. A brief one-paragraph instruction sheet was provided that paralleled the experimenter's spoken explanation of the task.

Procedure. The experimenter explained that the observers were to estimate the relative geographical areas of states (countries) from memory. Pennsylvania (France) had been previously selected for use as a standard since it is familiar and near the median of the size range to be estimated. It was explained that the area of Pennsylvania (France) was to serve as a standard and that it should be assigned a value of 100 units. The observers were told to assign numbers to each of the states (countries) such that the ratio of the estimate to 100 reflected the ratio of the area of that state (country) to Pennsylvania (France). In other words, if the area seemed twice as large as the standard it should be assigned 200; if it seemed half as large it should be assigned 50; and so on. Observers were tested in groups during a class period, but worked independently at their own pace. No participant required more than 20 min to complete the task.

\section{Results}

Geometric mean estimates were computed for each state (country) by collapsing across all observers in each of the two groups. Since Equation 1 becomes linear in $\log \Phi$ and $\log \Psi$ coordinates with a slope equal to the exponent $n$, power functions may be easily fit to the observed data by standard linear regression techniques. Power functions were fit to the log mean estimates and log actual areas (square miles) for both the state and country data using the method of least squares.

Table 1

Power Function Exponents (n) and Correlations (r) for Memorial Estimates of Geographical Area (Experiment 1)

\begin{tabular}{cccccc}
\hline & \multicolumn{2}{c}{ Group Data } & & \multicolumn{2}{c}{ Median Individual Data } \\
& States & Countries & & States & Countries \\
\hline $\mathrm{n}$ & .40 & .31 & .37 & .32 \\
$\mathrm{r}$ & .96 & .88 & .74 & .68 \\
\hline
\end{tabular}


In addition, Pearson product-moment correlations were computed between log size estimates and $\log$ actual area to provide goodness-of-fit estimates. The exponents and correlations obtained using this procedure are displayed in Table 1 for both overall group data and representative (i.e., median) data from individual observers. The group data are plotted in Figure 1. Quite clearly, subjective size estimates from memory were systematically related to actual state or country size for both individual and group data. In addition, the power functions provided a reasonably good fit to the data as indicated by the relatively high correlations obtained. These findings are consistent with the preliminary memory-psychophysical studies described above and support the proposition that memory size scales have ratio properties in the same sense that perceptual size scales do.

\section{EXPERIMENT 2}

The findings of Experiment 1 are generally consistent with other data in revealing that: (1) memory size estimates are power functions of actual area (Bjorkman
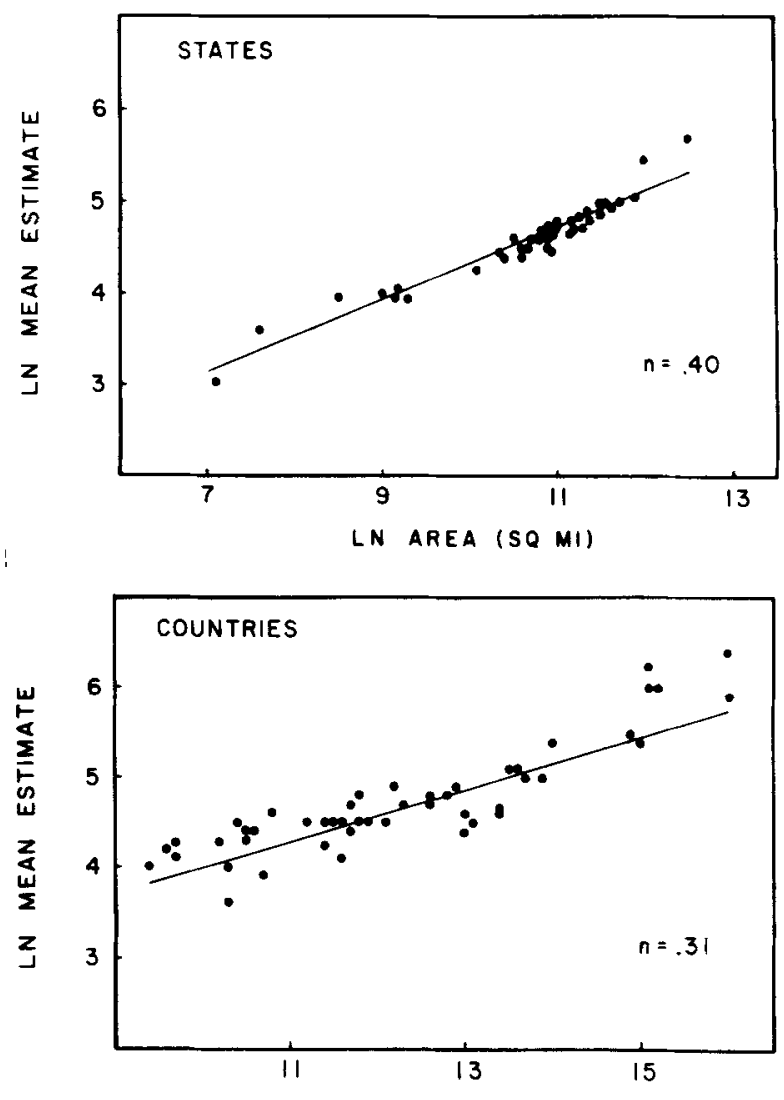

LN AREA (SO MI)

Figure 1. Log geometric mean magnitude estimates of area as a function of $\log$ actual area (square miles) for states and countries, Experiment 1. Linear regression lines and slopes (n) are shown. (Note-Overlapping data are plotted as a single point.) et al., 1960; Lundberg \& Ekman, 1971), and (2) memory-psychophysical exponents are somewhat smaller than those obtained for perceptual judgments of area (Moyer, Bradley, Sorensen, Whiting, \& Mansfield, 1978). Of further interest, however, are the actual magnitudes of the memory exponents and their relation to the magnitudes of the corresponding perceptual exponents. In the present experiment, the memory exponents $(.40$ and .31 for states and countries, respectively) are considerably smaller than those observed in earlier perceptual studies (e.g., .80, Teghtsoonian, 1965). Unfortunately, since perceptual size judgments were not obtained in the present experiment, the psychological basis of this discrepancy cannot be determined. It is possible, for example, that subjective judgments of highly familiar meaningful objects (e.g., states and countries) are characterized by different exponents than are the meaningless random shapes and stylized stimuli more typically investigated in the literature (e.g., Teghtsoonian, 1965). In addition, since observers in the present experiment were not explicitly familiarized with the objects to be compared, we cannot be certain that their initial learning of state and country area was based on accurate information.

In Experiment 2, subjective size estimates of state area were obtained under both perceptual and memorial conditions. In the perceptual condition observers made their judgments while viewing a map of the contiguous 48 states, while in the memorial condition observers judged relative state size from memory after having previously studied a map. If the exponents observed for the perceptual condition are consistent with those reported in the literature, then the relation between the actual magnitudes of the memory and perceptual exponents can be determined.

\section{Method}

Subjects. Twenty-one student volunteers participated in the perceptual group and 18 students participated in the memorial group. No participant had served in Experiment 1.

Materials. Observers recorded their size estimates of the 48 states of the continental United States on computer-generated sheets in a different random order for each participant. Answer spaces were provided for size estimates and for ratings of subjective confidence. Confidence estimates were rated on a scale from 1 (low) to 5 (high). An outline map (21.6 $27.9 \mathrm{~cm})$, showing only the boundaries and names of the states, was provided for all observers. A one-paragraph instruction sheet gave written instructions that were identical to the experimenter's spoken instructions.

Procedure. Observers in the perceptual group were provided copies of the materials and were read the following instructions: "Please rate the following states in terms of their geographical size or area. Assume that Pennsylvania is 100 units in size. If you think a state is twice as large as that, rate it 200 units. If you think it is two and a half times as large, rate it 250 units, and so on. If a state is half as big as Pennsylvania, you would rate it 50 units. Your ratings don't have to be in round numbers; feel free to use any number 'in between' that you feel describes the size of the state. When you make each rating, please indicate how confident you are of your judgment on a scale where: 
Table 2

Power Function Exponents (n) and Correlations (r) for Perceptual and Memorial Estimates of Geographical Area (Experiment 2)

\begin{tabular}{cccccc} 
& \multicolumn{2}{c}{ Group Data } & & \multicolumn{2}{c}{ Median Individual Data } \\
\cline { 2 - 3 } & Perception & Mernory & & Perception & Memory \\
\hline $\mathrm{n}$ & .79 & .60 & .80 & .59 \\
$\mathrm{I}$ & .99 & .97 & .97 & .89 \\
\hline
\end{tabular}

$5=$ very sure, $4=$ above average, $3=$ average, $2=$ unsure, $1=$ very unsure."

Maps and instructions were provided at the same time, and observers in the perceptual group made their area estimates while viewing a copy of the map at their desks. In the memorial group, maps were distributed first and observers were asked to study the map to get a good idea of the relative sizes of the states. A 7-min period was allowed for study. Following this interval, the experimenter collected the maps, distributed the answer sheets, and instructed the observers in the judgment task. The collection and distribution of materials and reading of the instructions required approximately $2 \mathrm{~min}$. The perceptual judgment group completed the entire task in about $20 \mathrm{~min}$, while the memory group required $30 \mathrm{~min}$.

\section{Results}

Size estimates. Geometric mean magnitude estimates were obtained for each state by collapsing across individual observer ratings within each group. Power functions were fit to individual observers' estimates as well as to the overall group means. Similarly, correlations were computed between $\log$ actual area and $\log$ estimates for both individual and group data. The results of these analyses are displayed in Table 2 for both group data and for representative individual data. Figure 2 displays a plot of the group data. As in Experiment 1, power functions provided a good fit to the data for both the perceptual and memory conditions, as indicated by the high correlation coefficients observed for both conditions $(r=.99$ and .97 for the perception and memory groups, respectively). In addition, the exponent observed for the perceptual condition $(n=.79)$ fell within the range expected from earlier studies (Macmillan, Moschetto, Bialostozky, \& Engel, 1974). However, despite this consistency, the exponent observed for the memory condition $(n=.60)$ was once again substantially smaller than that observed for the perceptual condition $[t(92)=5.603, p<.001]$. The fact that the perceptual exponent was generally consistent with earlier studies employing random, unfamiliar visual shapes rules out the possibility that highly familiar visual objects (i.e., outline drawings of states) are characterized by different exponents than are unfamiliar objects. In addition, the prefamiliarization procedure used in the present experiment insured that accurate information regarding state area was available to observers in the memory condition. These findings support the argument that memory-psychophysical exponents are smaller in magnitude than are their corresponding perceptual exponents, and that this difference cannot be attributed to any simple procedural artifact.

Confidence ratings. A subjective confidence rating was obtained for each state judgment for each individual in both groups. Three observers in the perceptual group either failed to indicate their confidence or indicated only a single confidence rating for all judgments. Confidence data from these observers were discarded. Confidence judgments for the remaining observers were submitted to a two-way analysis of variance (group by state) with repeated measurements on the state factor. Of central interest in this analysis was a possible difference in overall confidence for the perceptual and memory groups. Although mean confidence was slightly higher for the perceptual group (3.26 and 3.07 for the perceptual and memorial judgments, respectively), this difference did not approach statistical significance $[F(1,34)<1.0]$.

In addition to the above finding, a significant main effect of state $[F(47,1598)=7.26, p<.001]$ and a sig.
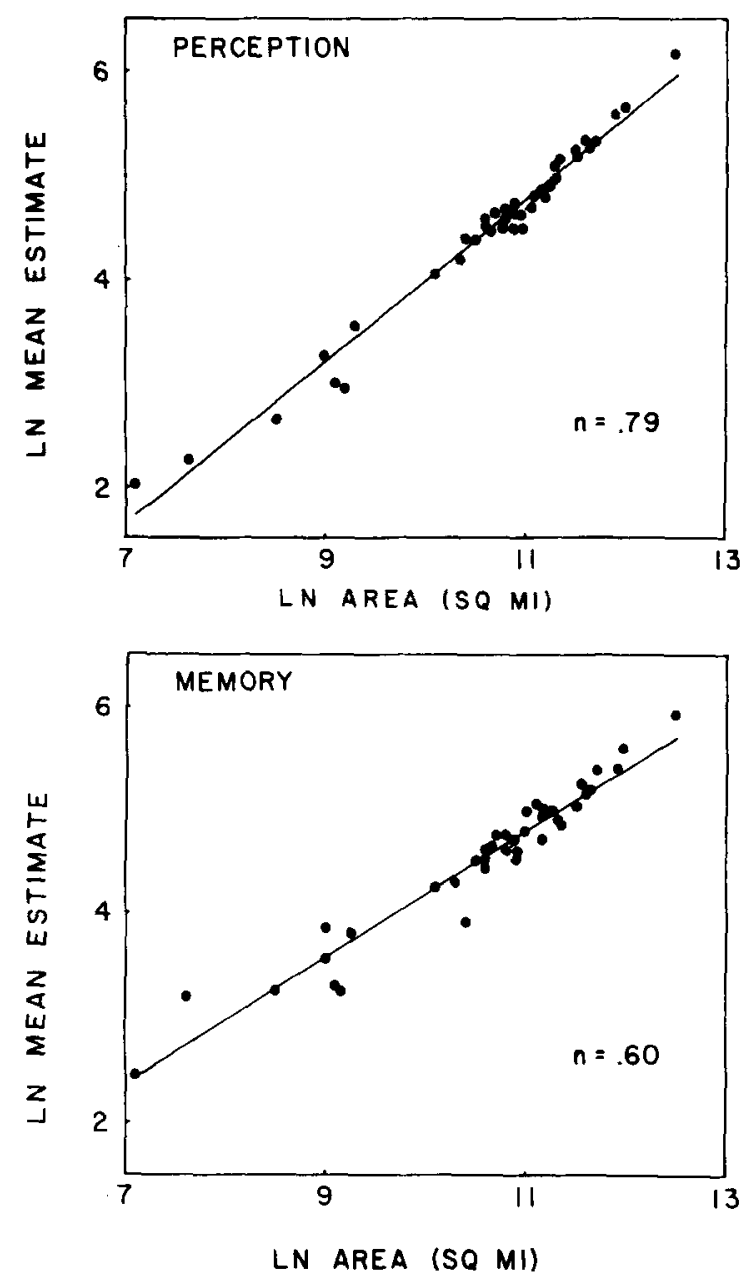

Figure 2. Log geometric mean magnitude estimates of state area as a function of actual area (square miles), Experiment 2. Linear regression lines and slopes (n) are shown. (Note-Overlapping data are plotted as a single point.) 
nificant State by Group interaction $[F(47,1598)=1.52$, $p<.05]$ were observed. The main effect of state was not unexpected given the exceptionally high confidence observed for the standard (Pennsylvania was 4.83 for both groups). Beyond this, no systematic trends were observed in the confidence data across the different states. In particular, the overall pattern of mean confidence ratings was highly similar for the two groups. The significant State by Group interaction can be attributed to the seven instances where higher mean confidence ratings were obtained for the memory group. It is interesting to note that of these seven cases, five were either local states (Delaware, Maryland, and Virginia) or the very smallest or largest of the states examined (Rhode Island and Texas).

\section{Discussion}

The results of the present experiment clearly indicate that for judgments of visual area, the memorial exponent is reliably smaller than the corresponding perceptual exponent. However, the psychological mechanism responsible for this effect remains unclear since two very different processes could account for the observed effect. The first possibility is that observers make size judgments from memory by directly "reading" some value or by sampling from some range of values that correspond to the area of each object. These stored area values would be expected to be similar to the perceptual magnitude estimates of visual area, particularly if the observer had seen the object recently. According to this hypothesis, observers in the memory condition experience greater uncertainty than perceptual observers and, hence, employ a more conservative range of magnitude estimates. This would result in a decrease in the value of the exponent (Teghtsoonian, 1971). A similar "response-constriction" effect has been discussed by Marks (1974) and by Stevens and Greenbaum (1966) in comparing data from magnitude estimation and production tasks.

An alternative possibility is that memorial size judgments are not based on stored values that are simply retrieved, but on visual images that are "re-perceived" when the judgments are made. Paivio (1975) has argued that continuous or analog (e.g., visual area) information about concrete objects and events is retained in a visual imagery mode. In his dual-coding theory (Paivio, 1971, 1974), the imagery and verbal systems are viewed as independent but connected domains for representing knowledge in memory. The verbal system deals with discrete linguistic representations, while the imagery system is more closely related to perceptual knowledge.

In the present re-perceptual model, a size judgment from memory is related to the actual size of an object by two separate transformations. In an initial perceptual transformation, the physical stimulus arriving at the receptors is processed or transformed into a perceptual experience. It is this transformation that yields the familiar power-function relation between physical magnitude and estimated psychological magnitude. This percept is then stored, in its transformed state, in long-term memory. At recall, a second re-perceptual transformation, very similar or identical to the initial perceptual transformation, is assumed to operate on the stored information to provide a subjective size estimate. According to this hypothesis, then, two power functions intervene between the actual physical magnitude of an object and its memorial size estimate. That is, for perception, the psychological magnitude, $\Psi_{p}$, is a power function of the actual physical magnitude, $\Phi ; \Psi_{\mathrm{p}}=\mathrm{k} \Phi^{\mathrm{n}}$. For memory, psychological magnitude, $\Psi_{\mathrm{m}}$, is a power function of the previously determined perceptual magnitude, $\Psi_{p} ; \Psi_{m}=k^{\prime} \Psi_{p}^{n^{\prime}}$. By substitution, it may be shown that memorial judgments are also power functions of the actual physical magnitudes: $\Psi_{m}=k^{\prime}\left(k \Phi^{n}\right)^{n^{\prime}}=K \Psi^{n \cdot n^{\prime}}=K \Phi^{N}$, where $\mathrm{K}$ is a new scaling factor to accommodate the memory estimates. Furthermore, since the two transformations are assumed to be identical, the memory exponent, $\mathrm{N}$, approximates the square of the perceptual exponent, $n$ (i.e., since $n=n^{\prime}, N=n^{2}$ ). This proposed square relation provides a reasonable description of the relation between the perceptual and memorial exponents observed in the present experiment $(.79$ and .60 , respectively).

Although Experiment 2 was not designed to distinguish between the uncertainty and re-perceptual hypotheses, the confidence data discussed above are relevant to this question. In particular, if range constriction due to uncertainty was the primary psychological mechanism responsible for the difference in perceptual and memorial exponents, one would also expect observers in the memory group to have lower confidence in their judgments than those in the perceptual group. As indicated above, a statistical treatment of the confidence data revealed no reliable difference between the two groups. Furthermore, the correlation coefficient for the memory group (.97) was virtually identical to that of the perceptual group (.99), indicating that the memory and perceptual data were equally systematic. While not conclusive, these findings suggest that an explanation based on range constriction is inadequate to account for the difference between perceptual and memorial exponents. Experiment 3 was designed to further clarify this issue.

\section{EXPERIMENT 3}

The present experiment was designed to test the uncertainty and re-perceptual hypotheses for perceptual and memorial judgments of visual length rather than area. Observers were asked to make perceptual and memorial estimates of the distances between the centers of the contiguous 48 states of the United States. 
The uncertainty and re-perceptual models predict different relations between the perceptual and memorial exponents in this situation. Consider first the uncertainty hypothesis. Since the memorial length judgments would be made under the same conditions as the area judgments of Experiment 2, the uncertainty model predicts that the memorial condition would yield a reduced range of subjective judgments and, hence, a smaller exponent than the perceptual condition. In other words, the same range-constriction processes should operate on subjective length estimates as operate on subjective area judgments. In contrast, the re-perceptual hypothesis predicts that exponents for the memorial and perceptual conditions should be identical. Since the exponent for perceptual judgments of length is typically close to unity (Baird, 1970), the re-perceptual hypothesis predicts that the memory exponent should be $1.0^{2}$ or 1.0 . If, in the present data, the memory exponent for length judgments were substantially less than 1.0 , then this finding would support the uncertainty hypothesis. However, if both exponents were equal to 1.0 , then the re-perceptual hypothesis would be supported.

\section{Method}

Subjects. Eleven student volunteers judged interstate distance from memory, and 12 rated the distances while viewing a map. No participant had served in either of the previous experiments.

Materials. Observers estimated the linear distance between the centers of 22 pairs of states sampled from the contiguous states of the United States. Each observer rated the same pairs, but in a different random order for each observer. Magnitude estimates and confidence ratings ( $1=$ low to $5=$ high $)$ were indicated on computer-generated response sheets. Order of presentation of each state within each pair was counterbalanced (i.e., North Dakota/Texas and Texas/North Dakota were presented with nearly equal frequency).

The 22 interstate distances to be tested were sampled by first obtaining visual estimates of the geographic centers of each of the 48 states from eight volunteer observers. Each volunteer simply indicated the geographic center on an outline map similar to that employed in Experiment 2. These estimates were then transferred to a master copy and were visually averaged by the experimenter to provide a "psychological center" for each state. The $x, y$ coordinates of each center were obtained by conventional graphic techniques and all 1,128 possible euclidean interstate distances were computed.

A pool of 48 distances that covered the range of north-south, east-west, and diagonal distances was selected as follows. Long distances were obtained by rotating a line about the geographic center of the United States and selecting the largest distances (i.e., border-to-border distances such as Maine/Arizona and North Dakota/Texas) which were near the line. Intermediate and short distances were obtained by successive north-south and east-west sweeps over the map for each distance range. Twenty-two distances were sampled from this pool, such that they covered a range of $1.18 \mathrm{log}$ units and were spaced approximately $.12 \mathrm{log}$ units apart. Distances between adjacent states were not used.

Procedure. Observers in the perceptual judgment condition made estimates of the 22 designated interstate distances while looking at a United States map at their desks. In the memory judgment condition, subjects were asked to study the map to get a good idea of the locations of the states. A period of $7 \mathrm{~min}$
Table 3

Power Function Exponents (n) and Correlations (r) for Perceptual and Memorial Estimates of Interstate Distances (Experiment 3)

\begin{tabular}{cccccc} 
& \multicolumn{2}{c}{ Group Data } & & \multicolumn{2}{c}{ Median Individual Data } \\
\cline { 2 - 3 } & Perception & Memory & & Perception & Memory \\
\hline $\mathrm{n}$ & 1.04 & 1.10 & & 1.02 & 1.08 \\
$\mathrm{r}$ & .98 & .96 & & .97 & .92 \\
\hline
\end{tabular}

was allowed for study. At the end of this interval, the maps were collected and subjects began to make distance estimates about $2 \mathrm{~min}$ after the experimenter read and distributed the following instructions and answer sheets. In the perceptual judgment group, these instructions were read by the experimenter and written copies were presented simultaneously with the maps:

"Please rate each of the following pairs of states in terms of the distance between the centers of the states. Assume that the distance from the middle of New York state to the middle of South Carolina is 100 units. If you think a given distance is twice as large as that, rate it 200 units. If you think it two and a half times as large, rate it 250 units, and so on. If a distance is half as big as the distance between the center of New York and the center of South Carolina, you would rate it 50 units. Your ratings don't have to be in round numbers; feel free to use any number 'in between' that you feel describes the distance between the states.

"When you make each rating, please indicate how confident you are of your judgment on a scale where: $5=$ very sure, $4=$ above average, $3=$ average, $2=$ unsure, and $1=$ very unsure."

\section{Results}

Distance estimates. Geometric mean magnitude estimates were obtained for each state pair by collapsing across individual distance estimates within each group. As in Experiments 1 and 2, power functions were fit to individual observer data as well as to the overall group means. These results are presented in Table 3, and the group data are plotted in Figure 3. Inspection of these findings reveals that power functions provided a reasonable description of the data for both groups $(r=.98$ and .96 for the perceptual and memorial groups, respectively). In addition, the exponent observed for the perceptual group $(n=1.04)$ was close to 1.0 and fell generally within the range of values observed in earlier studies of visual length (Stevens, 1975). More interesting in the present context is the finding that the memorial exponent $(n=1.10)$ was also consistent with earlier perceptual findings. In fact, the observation that the memorial exponent in the present study was slightly larger than the perceptual exponent $(n=1.04)$ would be predicted by the re-perceptual hypothesis (i.e., $1.04^{2}=1.08$ ). Most importantly, it is clear that the memorial exponent was not smaller than the perceptual exponent, as would be predicted by the alternative uncertainty hypothesis.

Confidence ratings. Subjective confidence judgments for each interstate distance judgment were submitted to a two-way analysis of variance (group by state pair) with repeated measures on the state pair factor. The 


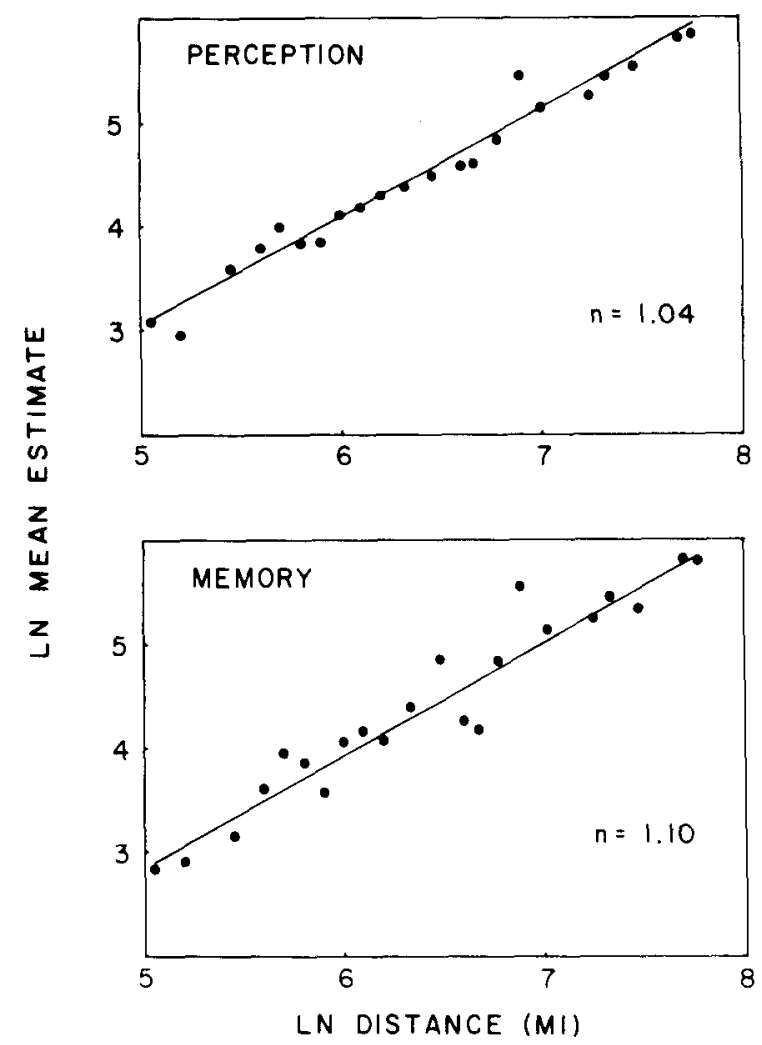

Figure 3. Log geometric mean magnitude estimates of interstate distance as a function of $\log$ actual distance (miles), Experiment 3. Linear regression lines and slopes (n) are shown.

findings paralleled those of Experiment 2. Mean confidence was slightly higher for the perceptual observers (3.59 and 3.32 for the perceptual and memorial groups, respectively), but this difference did not approach statistical significance $[F(1,20)=1.20$, $\mathrm{p}>.20]$.

In addition to this finding, a significant main effect of state pair $[F(21,420)=2.16, p<.001]$ and a significant State Pair by Group interaction $[F(21,420)=2.57$, $\mathrm{p}<.001]$ were observed. Figure 4 presents a plot of the mean confidence for each group and each state pair, ordered by increasing log distance along the abscissa. Visual inspection of these data reveals that for 14 of 22 state pairs, mean confidence was greater for the perceptual group than for the memorial group. The intermediate and moderately long distances revealed a consistently higher mean confidence for the perceptual group. However, six of the seven cases where this trend was reversed (i.e., where mean confidence was greater for the memorial group) occurred for the relatively short or extremely long interstate distances. This trend is similar to that reported in Experiment 2 for judgments of state area and would account for the reliable Group by State Pair interaction.

\section{Discussion}

The above findings offer clear support for the re-perceptual hypothesis. Both perceptual and memorial magnitude estimates of interstate distance were observed to be power functions of the actual distances. In addition, the exponent of the best-fitting power function for the memorial condition was slightly larger than the corresponding value observed for the perceptual condition. Since the perceptual exponent (1.04) was somewhat greater than 1.0 , the re-perceptual hypothesis would predict a slightly larger memorial exponent of 1.08. The observed memorial exponent, 1.10 , was well approximated by this value. In contrast, the uncertainty hypothesis predicts that the memorial exponent would be substantially smaller than the perceptual exponent, as observed in Experiment 2.

In addition, the subjective confidence data were also inconsistent with the uncertainty hypothesis. This analysis revealed a systematic tendency for memorial observers to have lower confidence in their judgments of intermediate and moderately long interstate distances than perceptual observers. For the two longest and six shortest distances, there was a tendency for this trend to reverse. Since the confidence judgments may be reasonably assumed to reflect observer uncertainty, these findings indicate that a slight increase in uncertainty occurred in the memorial judgment condition. In spite of this fact, the memory exponent was not smaller than the perceptual exponent.

\section{GENERAL DISCUSSION}

As Shepard and Podgorny (in press) point out in their recent review, much of the existing research on human memory has ignored or overlooked a number of impor tant similarities between memorial and perceptual processes. They suggest that the ultimate goal of developing a general theory of cognition may be better served if future work were to investigate these similarities. The present study, as the work of Moyer (1973; Moyer \& Bayer, 1976; Moyer et al., Note 1) and others (e.g.,

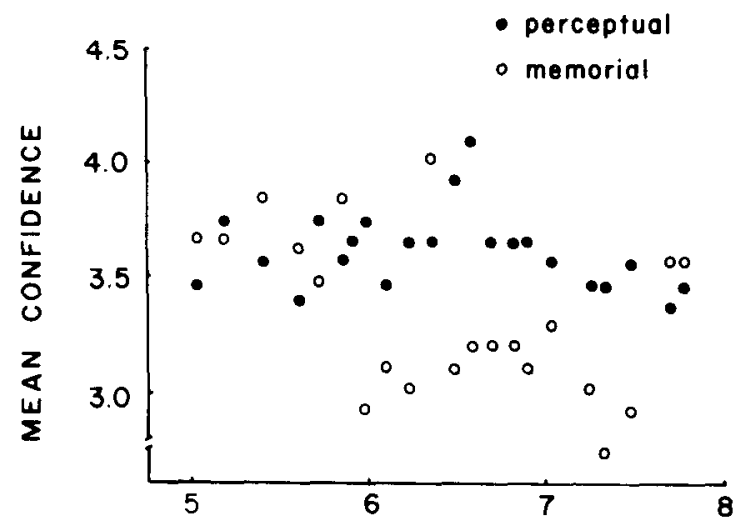

LN DISTANCE (MILES)

Figure 4. Mean confidence ratings for interstate distance estimates, plotted as a function of log distance. 
Kerst \& Howard, 1977; Paivio, 1975), was undertaken in this spirit. The primary purpose of the present study was to investigate the underlying properties of the memory code for visual area and length in a simple judgment task. How fine grained is the code? How does our remembered knowledge of these dimensions compare to our perceptual experience of them? In psychophysical terms, these questions concern the nature of the memory-psychophysical scale and its relation to the perceptual scale. The present study has applied existing psychophysical method and theory to the investigation of both issues.

First, consider the underlying characteristics of the memory code. The results of the present study indicate that memorial estimates of visual area and length, like perceptual estimates of these attributes, are power functions of their corresponding physical values. In addition, the relation between the memorial exponents for area and length judgments paralleled the relation between the perceptual exponents for these continua. For both memorial and perceptual conditions, area exponents were substantially smaller than length exponents. The occurrence of distinct memory exponents for the two continua suggests that the memory-psychophysical scale for these attributes has more than ordinal properties (e.g., Stevens, 1975). If only rank-order information had been available to observers in the memory condition, then extremely similar memory exponents would be expected for the two continua investigated in the present study. This interpretation is consistent with earlier theory that advocates a precise, continuous basis for the memory representation of "visual" information (e.g., Moyer, 1973; Paivio, 1974).

The second major issue addressed by the present study concerns the relation between the underlying memory scale and its corresponding perceptual scale. In the Moyer et al. (1978) investigation of remembered size and in the present investigation of remembered area, memory exponents were observed to be considerably smaller than the corresponding perceptual exponents. At first glance, this finding suggests that for memory judgments, uncertainty or forgetting-with subsequent guessing-serves to reduce the range of subjective estimates and, hence, reduces the magnitude of the exponent. However, the results of Experiment 3 indicate that the relation between memorial and perceptual exponents depends on the magnitude of the perceptual exponent. Memorial exponents are not always smaller than their corresponding perceptual exponents, rather they appear to approximate the square of the perceptual exponent. As indicated above, this relation would be expected if memorial judgments of perceptual continua result from a re-perceptual process that operates on stored perceptual representations. According to this model, an initial power-function transformation operates on the sensory input to produce a perceptual represen- tation. It is this initial transformation that determines the psychophysical power law discussed by Stevens and many others (e.g., Marks, 1974; Stevens, 1975). This perceptual representation is then coded into long-term memory for later use. In a subsequent task where observers are required to make psychophysical judgments from memory, the perceptual transformation is applied to the stored data to re-perceive the original stimulus. Although this re-perceptual model is preliminary, it appears reasonable in light of a number of considerations. First, the square relation between memorial and perceptual exponents was observed in Experiments 2 and 3 of the present study. Second, the square relation predicted by the model is also consistent with the Moyer et al. (1978) data on remembered area. In that study Moyer reported perceptual exponents of .643 and .729 for area and volume estimates of two- and three-dimensional stimuli, respectively. In both cases the observed memorial exponent (.463 and .527) was approximated by the square of the perceptual exponent (.413 and .531 , respectively). ${ }^{3}$ Third, preliminary findings indicate that the square relation is obtained only for extensive judgment continua such as visual area and volume. The Moyer et al. (Note 1) data on brightness (memory exponent $=.487$, perceptual exponent $=.461$ ) and preliminary data from our laboratory on loudness memory (memory exponent $=.253$, perceptual exponent $=.238$ ) suggest that the square relation is not observed for intensive continua. In this context, it is important to note that the power functions that characterize many intensive continua have been attributed largely to sensory or transducer processes in the receptor organ itself (e.g., Stevens, 1970). Since it would be unreasonable to assume that the receptors are involved in the re-perceptual process, one would not predict that memory would introduce a second power transformation identical in magnitude to the perceptual one. Hence, the re-perceptual hypothesis would not predict that memory exponents would be the square of perceptual exponents for intensive continua. Fourth, the proposed re-perceptual model is compatible with existing theoretical work on human visual memory. For example, Kosslyn (1975) provides a particularly explicit metaphor to characterize mental images that is related to the present re-perceptual hypothesis. His approach is constructivist in that visual images are thought to be generated from abstract units stored in long-term memory and based on perceptual experience. These abstract memory codes are the products of perception, but they are not directly available. Rather, they are acted on by constructive processes that produce the subjective experience of a mental image. In Kosslyn's theory, the relation between the mental image and the abstract perceptual units from which it is constructed is similar to the relation between the display on a CRT screen and the computer program used to generate 
the display. The visual display (mental image) but not the program (abstract perceptual units) can be seen by the viewer. In terms of Kosslyn's model, the present re-perceptual hypothesis assumes that the constructive process involved in the recall of a mental image is similar to the process underlying the initial perceptual experience of the stimulus. When this process is reapplied to "inspect" a mental image from memory, the image, which already reflects the original perceptual transformation, undergoes an additional transformation.

Despite the apparent consistency of the proposed re-perceptual model, a more complete statement of the relation between perceptual and memorial processes must await additional empirical evidence. Areas of future research should include an extension of the present methods to other judgment continua characterized by distinct perceptual exponents, as well as an investigation of the differences between intensive and extensive dimensions. Nonetheless, the results of the present study, as well as Moyer et al.'s findings (1978, Note 1) suggest that psychophysical methods and theory can be productively applied to the analysis of complex memory phenomena.

\section{REFERENCE NOTES}

1. Moyer. R. S., Bradley, D. R., \& Cutcomb, S. Memory psychophysics. Paper presented at the annual meeting of the Eastern Psychological Association, Boston, April 1977.

\section{REFERENCES}

BaIro. J. C. A psychophysical analysis of visual space. Oxford: Pergamon Press, 1970.

Banks, W. P. Encoding and processing of symbolic information in comparative judgments. In $G$. $H$. Bower (Ed.), The psychology of leaming and motivation (Vol. 11). In press.

Björkman, M., LundberG, I., \& TÄrnblom, S. On the relationship between percept and memory: A psychophysical approach. Scandinavian Journal of Psychology. $1960,1.136-144$.

Curtis, D. W., Paulos, M. A., \& Rule, S. J. Relation between disjunctive reaction time and stimulus difference. Journal of Experimental Psychology, 1973, 99, 167-173.

Jamieson, D. G., \& Petrusic, W. M. Relational judgments with remembered stimuli. Perception \& Psychophysics, 1975, 18, 373-378.

KeRST, S. M., \& Howard, J. H., JR. Mental comparisons for ordered information on abstract and concrete dimensions. Memory \& Cognition, 1977, 5, 227-234.

KossLyn, S. M. Information representation in visual images. Cognitive Psychology, 1975, 7, 341-370.

LuCE, R. D. On the possible psychophysical laws. Psychological Review, 1959, 66, 81-95.

LUNDBERG, V., \& Ekman, G. Geographical data as psychophysical stimuli. Scandinavian Joumal of Psychology, 1972, 13, $81-88$.

Macmillan, N. A., Moschetto, C. F., Bialostozky, F. M., \& ENGEL, L. Size judgment: The presence of a stan- dard increases the exponent of the power law. Perception \& Psychophysics, 1974, 16. 340-346.

Marks, L. E. Sensory processes: The new psychophysics. New York: Academic Press, 1974.

MOYer. R. S. Comparing objects in memory: Evidence suggesting an internal psychophysics. Perception \& Psychophysics, 1973, 13, 180-184.

Moyer. R. S., \& BAyer, R. H. Mental comparison and the symbolic distance effect. Cognitive Psychology, 1976, 8, 228-246.

Moyer, R. S., Bradley, D. R., Sorensen, M. H., Whiting, J. C., \& Mansfield, D. P. Psychophysical functions for perceived and remembered size. Science, 1978, 200, 330-332.

Moyer, R. S. \& DumaIs, S. T. Mental comparison. In G. H. Bower (Ed.). The psychology of learning and motivation (Vol. 12). In press.

Parvio, A. Imagen and verbal processes. New York: Holt, Rinehart, \& Winston, 1971.

PAivio. A. Language and knowledge of the world. Educational Researcher. 1974, 3, 5-12.

Paivio. A. Perceptual comparisons through the mind's eye. Memory \& Cognition, 1975, 3, 635-647.

Ports, G. R. Integrating new and old information. Journal of Verbal Learning and Verbal Behavior, 1977, 16, 305-320.

ShePARd, R. N.. \& Podgorny, P. Cognitive processes that resemble perceptual processes. In W. K. Estes (Ed.), Handbook of learning and cognitive processes. Hillsdale, N.J: Lawrence Erlbaum, in press.

Stevens, S. S. Neural events and the psychophysical law. Science, 1970, 170, 1043-1050.

Stevens, S. S. Psychophysics: Introduction to its perceptual. neural and social prospects. New York: Wiley, 1975.

Stevens, S. S., \& Greenbaum, H. B. Regression effect in psychophysical judgment. Perception \& Psychophysics, $1966,1.437-446$.

Teghtsoonian, M. The judgment of size. American Journal of Psychology, 1965. 78, 392-402.

Teghtsoonian, R. On the exponents in Steven's law and the constant in Ekman's law. Psychological Review, 1971, 78. $71-80$.

\section{NOTES}

1. Although Banks (in press) was unable to replicate Moyer and Bayer's results regarding the effects of absolute size, Potts (1977) has reported results consistent with Moyer and Bayer.

2. The work reported here was begun in August 1976. We learned of Moyer's similar but independent work in April 1977.

3. In an additional study, Moyer et al. (1978) have observed perceptual and memorial exponents of .867 and .703 , respectively, for judgments of line length. The unexpectedly low perceptual exponent may be due to the absence of a standard in his judgment task (Macmillan et al., 1974). In addition, since Moyer employed a 24-h retention interval between learning and test, forgetting and cautious judgment may have caused a decrease in the memorial exponent beyond that predicted by the re-perceptual model $\left(.867^{2}=.752\right)$. Nevertheless, these results and his data on area judgments led Moyer et al. (1978) to suggest a model similar to the one independently developed and tested in the present paper.

(Received for publication October 19, 1977; revision accepted March 7, 1978.) 